\title{
Fellow Column: COVID-19: The Latest TORCH Infection?
}

Sarin Pakhdikian, OMS III

The placenta is the primary site of nutrient and gas exchange between the mother and fetus. The two components, fetal and maternal, are made of parts that confer growth and resilience. Cytotrophoblasts are the inner layer of the chorionic villi that make up the cells, while the syncytiotrophoblasts are the outer layer responsible for synthesizing and secreting hormones (such as beta-human chorionic gonadotrophin). It lacks MHC-I expression, which decreases the chance of attack by the maternal immune system. (1) The decidua basalis makes up the maternal component, derived from the endometrium, and stores maternal blood in the lacunae.

Select microbes are notorious for bypassing the protective barriers from mother to fetus via transplacental transmission or during delivery. Toxoplasma gondii poses the threat of chorioretinitis, hydrocephalus, and intracranial calcifications. Rubella can lead to cataracts, deafness, and congenital heart disease (i.e., patient ductus arteriosus), while cytomegalovirus can cause hearing loss, seizures, petechial rash, and periventricular calcifications. Human immunodeficiency virus, which can be well managed during pregnancy, may give rise to chronic infections and diarrhea in the infant. Herpes simplex virus- 2 may result in meningoencephalitis or herpetic vesicular lesions. Syphilis can cause stillbirth and hydrops fetalis.

It is now important for us to consider where this leaves COVID-19 infections.

\section{"Select microbes are notorious for bypassing the protective barriers from mother to fetus via transplacental transmission or during delivery."}

In a review by Karimi-Zarchi, et al. (2020), the authors speak to the vertical transmission of coronavirus from infected mothers to neonates. In the case of 31 infected mothers with COVID, there was no indication of COVID-19 nucleic acid detected in the placentas or neonatal throat swabs by RT-PCR. (2) There were no specific recommendations, however, to pregnant women regarding the evaluation or management of COVID, which is important considering that the transmission of the virus via respiratory droplets during breastfeeding has been documented. Two mothers had died of COVID related respiratory issues after delivery. Their clinical characteristics were similar to non-pregnant women with the infection. Maternal pneumonia was related to adverse obstetrical outcomes such as preterm rupture of membranes, preterm labor, intrauterine fetal demise, intrauterine growth restriction.

The clinical characteristics of coronavirus disease in newborns, infants, and children were discussed by Hong et al. (2020). The first critically ill patient reported at Wuhan Children's Hospital was a 1-year old male. He presented with intermittent diarrhea and vomiting for six days, fever, and shortness of breath. Pneumonia was evident in the right lung on the day of admission. The patient did not test positive for the virus until day eight and was weaned off the ventilator by day 10 , fully recovering from the virus. Pediatric cases were caused mainly by family clusters. These infections were epidemiologically linked to adult patients, with at least one infected family member in the household. (3) The youngest case for a neonatal infection was noted 30 hours after birth with no evidence of vertical transmission.

\section{"These infections were epidemiologically linked to adult patients, with at least one infected family member in the household. (3) The youngest case for a neonatal infection was noted 30 hours after birth with no evidence of vertical transmission."}

There has been a debate on whether SARS-COV-2 is the newest spark in the TORCH infections. Muldoon et al. (2020) discuss infections during pregnancy are increasingly being described, but the frequency and severity of infections in newborns are incompletely defined. In a sample of infected fetal mice during all three trimesters of pregnancy, no fetal infection was noted, along with a variation among different strains of mice. Infection of cats with the coronavirus feline infectious peritonitis virus resulted in newborn kittens becoming carriers of the virus. There was no conclusive evidence of transplacental transfer of SARS-COV-2 from mothers with COVID-19 disease. Signs and symptoms of the illness in infants and infected mothers were noted as shortness of breath, fever, thrombocytopenia, abnormal liver function, tachycardia, vomiting, and pneumothorax. In a case series of six women with mild COVID-19 disease, the authors discuss the multiples measures taken to perform a Caesarean section, including isolation of their infants following delivery, no delay in cord clamping, and reduced infant to breast directly after delivery. (4) Two of these six infants had IgM antibodies to SARS-COV-2 present, although neither had symptoms. All of these infants repeatedly tested negative when tested for viral RNA upon subsequent testing. Another analysis of 33 neonates born to mothers with COVID-19 identified three neonates with SARS-COV-2 infection. Positive IgM serology was found in a neonate born by Cesarean section to a mother with SARS-COV-2 pneumonia. Current data suggest that IgM antibodies against the virus develop in the circulation after the first week of infection, while IgG antibodies become detectable sometimes beyond 14 days of infection. As a result, the detection of specific antibodies (IgM, IgG, or both) would suggest that a person has been infected with the virus at some point in time.

In a recent case report by Sisman et al. (2020), a woman experienced a complicated pregnancy due to maternal diabetes and obesity, late-latent syphilis, and preterm premature rupture of membranes. The infant was born at 34 weeks' gestation, large for gestational age, and admitted to the NICU. The problem list included prematurity, glucose monitoring, and SARS-CoV-2 exposure. Within 24 hours, the infant experienced fevers and respiratory distress, testing positive at 24 and 48 hours of life for the 
virus. Interventions began with ampicillin, gentamicin, and acyclovir. The patient was weaned to room air by day five, although still testing positive on day 14 . Upon discharge, on day 21 , the infant tested negative and had fully recovered. The most prominent finding, in this case, was the presence of cytoplasmic staining for the SARS-CoV-2 nucleocapsid protein by immunohistochemistry and demonstration of viral particles by electron microscopy in the syncytiotrophoblastic cells, strongly suggesting in utero transmission. (5)

\section{"It is important to explore how}

\section{transmission of this virus may occur due to ascending infection with} premature rupture of membranes and primary involvement of the maternal gastrointestinal tract, or by hematogenous spread if the mother was viremic during her initial infectious period."

It is important to explore how transmission of this virus may occur due to ascending infection with premature rupture of membranes and primary involvement of the maternal gastrointestinal tract, or by hematogenous spread if the mother was viremic during her initial infectious period. Further case studies and reviews are needed to determine the risks of vaginal delivery of mothers with SARS-CoV-2. More robust pre and postpartum prevention guidelines should also be established to test amniotic fluid, breastmilk, PCR techniques, as well as cord blood antibody testing.

\section{References:}

1. Le, T., Bhushan, V., Sochat, M., Schimansky, S., Kallianos, K., Vaidyanathan, V., and Abrams, J., n.d. First Aid For The USMLE Step 12020.

2. Karimi-Zarchi, M., Neamatzadeh, H., Dastgheib, S., Abbasi, H., Mirjalili, S., Behforouz, A., Ferdosian, F., and Bahrami, $R .$, 2020. Vertical Transmission of Coronavirus Disease 19 (COVID-19) from Infected Pregnant Mothers to Neonates: $A$ Review. Fetal and Pediatric Pathology, 39(3), pp.246-250.

3. Hong, H., Wang, Y., Chung, H., and Chen, C., 2020. Clinical characteristics of novel coronavirus disease 2019 (COVID-19) in newborns, infants, and children. Pediatrics \& Neonatology, 61(2), pp.131-132.

4. Muldoon, K., Fowler, K., Pesch, M., and Schleiss, M., 2020. SARS-CoV-2: Is it the newest spark in the TORCH?. Journal of Clinical Virology, 127, p.104372.

5. Sisman, J., Jaleel, M., Moreno, W., Rajaram, V., Collins, R., Savani, R., Rakheja, D., and Evans, A., 2020. INTRAUTERINE TRANSMISSION OF SARS-COV-2 INFECTION IN A PRETERM INFANT. Pediatric Infectious Disease Journal, Publish Ahead of Print.

Disclosure: The author identifies no conflict of interest

NT

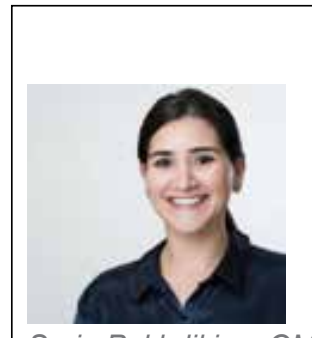

Sarin Pakhdikian, OMS III

Third Year Medical Student

Western University of Health Sciences

Pomona, CA

Sarin Pakhdikian <sarin.pakhdikian@westernu.edu>

Fellow's Column is published monthly.

- Submission guidelines for "Fellow's Column":

- 2000 word limit not including references or title page. Exceptions will be made on a case by case basis

- $\quad$ QI/QA work, case studies, or a poster from a scientific meeting may be submitted..

- Submission should be from a resident, fellow, or NNP in training.

- Topics may include Perinatology, Neonatology, and Younger Pediatric patients.

- No more than 20 references.

- Please send your submissions to:

Elba Fayard, MD

Interim Fellowship Column Editor LomaLindaPublishingCompany@gmail.com

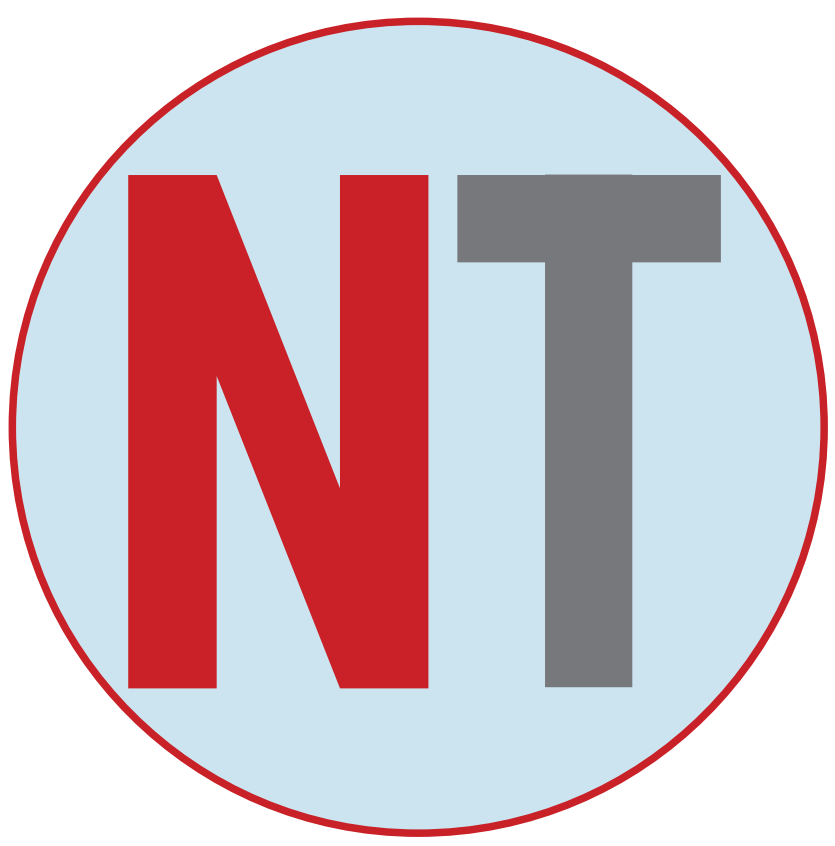

